\title{
RANDOMIZED CONTROLLED STUDY FOR EVALUATION OF BIODENTINE AND ENDOCEM ZR AS A TREATMENT OF REVERSIBLE PULPITIS IN MATURE PERMANENT TEETH ON PEDIATRIC PATIENTS
}

\author{
Nihal Kabel ${ }^{*}$ and Ghada Salem**
}

\begin{abstract}
Aim The aim of this study was to evaluate and compare the clinical outcomes of vital pulpotomy using biodentine (Septodent, France) or Endocem (Maruchi, Wonju, Korea) as pulp capping materials in a prospective randomized controlled study.
\end{abstract}

Materials and Methods This study was conducted in 20 subjects who were recruited from patients of the Department of pediatric dentistry of Misr University For science and Technology, Cairo, Egypt. All the patients in this study (10-13 year) were suffering from reversible pulpities in their mature permanent molars, a total of 40 teeth were randomly assigned to either the Biodentin or the Endocem group (20 teeth per group). Vital pulpotomy was done using these 2 materials, and clinical and radiographic evaluations were performed at 6 weaks, 6 months, and 1 year follow up period. Teeth with negative response to vitality test and those who exhibit signs of irreversible pulpitis or pulp necrosis were considered as failures.

Results In the 6 weeks, 6months and 1 year follow up, the clinical success rates for the ENDOCEM Zr was $95 \% .90 \%$ and $90 \%$, respectively and the radiographic success rates were $100 \%, 90 \%$ and $90 \%$ respectively. Regarding biodentine pulpotomy, the clinical and radiographic success rate in 6 weeks, 6months and 1 year follow up was $100 \%, 95 \%$ and $95 \%$ respectively. The difference between biodentine and MTA groups was statistically non-significant $(p=0.7646>0.05)$.

Conclusions in this randomized controlled study, Biodentine has clinical and radiograghic success rate comparable to ENDOCEM $\mathrm{Zr}$ as a vital pulpotomy in mature permanent molars with carious exposures.

KEY WORDS: Vital pulpotomy, Biodentine, ENDOCEM Zr.

* Lecturer, Department of Pediatric dentistry, faculty of Oral and Dental Medicine, MUST University.

** Researcher, Orthodontic and Pedodontic Department, National Research Center. 


\section{INTRODUCTION}

A principal challenge faced by most pediatric dentists is the treatment of pulpally involved teeth in young patients. Root canal treatment is the ideal treatment for permanent teeth with closed apex in pediatric patients but it is a complex costly procedure requiring prolonged appointments, cooperation of the patient and multiple visits and often requires a full coverage restoration ${ }^{1}$. With the development in understanding of pulp tissue regeneration and the development of new materials and technical use, attention was given to study partial pulp tissue regeneration to maintain pulp vitality using minimal invasive endodontic therapies ${ }^{2}$. Vital pulpotomy provide an alternative procedure to the conventional RCT in vital permanent teeth with carious exposed pulp in children. As the younger pulps had better tissue healing and regeneration related to elevation of cell populations in the pulp, this increase the success rate of vital pulpotomy in permanent teeth in children ${ }^{3}$.

Compared to RCT, vital pulpotomy is less technique sensitive especially for treating of posterior permanent teeth. Hence, vital pulpotomy may increase accessibility to dental care and more clinicians would be able to provide this affordable procedure when the patient's finances prevent them from doing RCT, leaving only extraction as a treatment option ${ }^{4}$. Also preservation of vital pulp allow for increasing the protective resistance of masticatory forces when compared to root canal treatment as the later may increases susceptibility to fracture due to loss of coronal and apical tooth structure $^{5,6}$.

Several materials used as a capping material, but there is yet no consensus on the ideal capping material. It is universally accepted that the ideal capping material should be biocompatible, antibacterial, have an effective seal and stimulate hard tissue regeneration ${ }^{7}$.

In the 1990s, Torabinejad at Loma Linda University developed a root-end filling material called Mineral trioxide aggregate (MTA $)^{8}$, which is a fine hydrophilic powder made up of tricalcium silicate, tricalcium aluminate, silicate oxide and tricalcium oxide 9 .

It has many advantages compared to other conventional capping materials such as its excellent sealing ability, biocompatibility, dentin bridge formation in cases of pulp capping and pulpotomy, antimicrobial properties and excellent marginal adaptation $^{6,10,11}$. So mineral trioxide aggregates (MTA) is accepted as the material of choice for vital pulp therapy in primary and young permanent teeth ${ }^{12}$. But it has been documented that MTA have many drawbacks. It includes discoloration potential (GMTA), difficult handling characteristics, Presence of toxic elements in the material composition (arsenic), expanded setting time, a non-predictable antimicrobial activity, high material cost, difficulty in removal after it is set and calcification of pulp chamber and root canal ${ }^{5,6,9,13}$. MTA contain bismuth oxide as a radiopacifier which may increase the cytotoxicity of MTA, because bismuth oxide does not encourage cell proliferation in cell culture ${ }^{8}$. This rendered this technique as a difficult and sensitive procedure, and restricted their use to specialists.

ENDOCEM $\mathrm{Zr}$ is a new generation of white MTA which has similar physical and biocompatible properties of the original MTA with the advantage of minimal discoloration that enhances a better esthetics, it also exhibits low grade of calcification of pulp chambers and root canals. The ENDOCEM $\mathrm{Zr}$ contains zirconia oxide instead of bismuth oxide. With the zirconia oxide, the subject device has higher Radiopacity ${ }^{14}$.

In 2009, synthetic tricalcium silicate cement (Biodentine) became commercially available ${ }^{15}$. It was initially and specifically designed as a "dentin replacement" material. Biodentine is initially formulated using the MTA-based cement technology with improvement in some properties. It is biocompatible and bioactive material ${ }^{16}$. Biodentine has 
several advantages which include good sealing ability, adequate compressive strength, and short setting time, which provide a significant clinical advantage over other comparable materials ${ }^{17}$.

However, a limited number of studies report the outcome of vital pulpotomy for mature permanent teeth in children and adolescents using either MTA or biodentine. Therefore the purpose of the present study is to assess and compare the clinical and radiographic outcome of vital pulpotomy for mature permanent teeth in children and adolescents using MTA and biodentine as a capping material.

\section{MATERIAL AND METHOD}

This study was conducted at the outpatient clinic of Pedodontic Department, Faculty of Oral and Dental Medicine, MUST University. Prior to examination, informed written consents for treatment and radiographs were taken from parents of all participating children after an explanation of the possible complications of the procedure. To be considered for this study, 20 patients were required to be medically healthy, have a restorable mature permanent molar with deep caries and a history of pain indicative of reversible pulpitis. Pain indicative of reversible pulpitis was defined as provoked short-duration pain that lasted for a few seconds and disappeared after removal of the stimulus, which could be reproduced using cold testing (Endo-Ice, Hygenic Corp, Akron, OH). Radiographic inclusion criteria included: teeth with closed apecies and normal periapical structures.

Exclusion criteria included patients with history of severe pain, history of swelling or a fistula associated with the tooth, tenderness on percussion or palpation, pathologic mobility, an abnormal response to cold testing or present of any periapical region in the radiogragh.

Patients were divided randomly into two groups; Group I: include 10 patients with 20 molars (right and left first permanent molar in each patient) received MTA pulpotomy and Group II: include 10 patients with 20 molars received Biodentine ${ }^{\mathrm{TM}}$ pulpotomy.

\section{Treatment procedure}

After anaesthetizing and isolating the tooth when possible using a rubber dam, the crown was disinfected with $5 \% \mathrm{NaOCl}$ before caries excavation, then caries was removed using a large round, low-speed carbide bur (Gebr.Brasseler1, Germany). Then standard pulpotomy procedure was performed, removing the infected coronal pulp tissue to the level of the floor of the pulp chamber and orifices by using a high-speed diamond bur (Gebr.Brasseler1, Germany) with copious sterile water. Pulp vitality was confirmed by the presence of bleeding pulp tissue from all canals. A sterile cotton pellet damped in $2.5 \% \mathrm{NaOCl}$ for $2 \mathrm{~min}$ was used to control the bleeding, and high pressure was applied. When the cotton pellets were removed homeostasis was apparent.

\section{In group I}

A layer of white MTA (ENDOCEM Zr), was mixed according to the manufacturer's instructions and was condensed with sterile cotton pellet on the floor of the pulp chamber and the root canal orifices with a thickness of approximately 2-3 mm. A damped cotton pellet was then covered with a temporary filling(IRM1, type III, Class 1 Caulk Dentsply, USA) as an intermediate restorative material till the next appointment in 2-7 days.

\section{In group II}

Biodentine was prepared according to the manufacturer's instruction by mixing a single-unit powder part to 5 drops of a single unit liquid part for 30 seconds. The mixture of Biodentine ${ }^{\mathrm{TM}}$ was then introduced into the pulp chamber using amalgam carrier to a thickness of approximately $3 \mathrm{~mm}$. and the procedure was completed as before. 
In the second visit, the cotton pellet and IRM were removed and the MTA and biodentine was checked to confirm setting using a small rounded burnisher. The set MTA and biodentine was then covered with resin-modified glass-ionomer cement (Vitrebond ${ }^{\mathrm{TM}}$, $3 \mathrm{M}^{\mathrm{TM}}$, ESPE, St. Paul, MN, USA) and restored using resin composite. All patients were scheduled for routine clinical and radiographic evaluations in 6weaks, 6 months and 1 year postoperatively. At each follow-up visit, the treated tooth was examined for any clinical signs and symptoms of pain, swelling, sinus tract, tenderness to percussion while evidence of periapical or furcation pathosis or root resorption were evaluated with periapical radiographs. All patients were asked whether they feel any spontaneous pain, pain on chewing or pain stimulated by hot/cold.
Pulp therapy was considered successful if none of the previous symptoms were present. The quality of the coronal restoration was checked, and the restoration was repaired if necessary.

An examiner who was not involved in the treatment phase of this study and who was previously calibrated, evaluate blindly all the pre and post radiographs at the end of the study. Data analysis was performed in several steps. Initially, frequent distribution for each group results was expressed in \%. Chi square test was performed to detect interaction between variables. Statistical analysis was performed using Aasistat 7.6 statistics software for Windows (Campina Grande, Paraiba state, Brazil). $\mathrm{P}$ values $\leq 0.05$ are considered to be statistically significant in all tests.

\begin{tabular}{|c|c|c|c|}
\hline Material & Components & Batch number & Manufacturers \\
\hline ENDOCEM Zr & $\begin{array}{l}\text { Tri-calcium silicate } \\
\text { Di-calcium silicate } \\
\text { Tri-calcium aluminate } \\
\text { Tetra-calcium-alumino-ferrite } \\
\text { Calcium sulfate dihydrate } \\
\text {-Zirconium Oxide }\end{array}$ & ZE8805110819 & Maruchi,onju, Korea \\
\hline $\begin{array}{l}\text { 3M Filtek }{ }^{\mathrm{TM}} \text { Z } 250 \\
\text { XT }\end{array}$ & Nanohybrid composite & N535294 & $\begin{array}{l}\text { 3M ESPE } \\
\text { Dental Products } \\
\text { St Paul, MN, USA }\end{array}$ \\
\hline Biodentine & $\begin{array}{l}\text { Powder } \\
\text {-Tricalcium Silicate. } \\
\text {-Dicalcium Silicate. } \\
\text {-Calcium Carbonate. } \\
\text {-Oxide filler. } \\
\text {-Iron Oxide shade. } \\
\text {-Zirconium Oxide. } \\
\text { Liquid } \\
\text {-Calcium chloride (accelerator). } \\
\text { Hydrosoluble polymer. }\end{array}$ & B02282 & Septodont \\
\hline
\end{tabular}




\section{RESULT}

All the twenty patients included in the study returned back for recall visits, Table 2 and 3 summarizes the distribution of teeth and the clinical and radiographic findings at the follow up periods of 6 weaks, 6 months, and 1 year in both Biodentine and MTA groups Figure (1) and (2). In Biodentine group, treatment was considered successful for 19 teeth $(95 \%)$. Only one tooth has been failed after six month of pulpotomy with pain, tenderness to touch and apical periodontitis. This case experienced loss of the permanent restoration during the follow up period. In MTA group, 18 teeth (90\%) were considered successful with two failures. The first case failed at 6 weeks as the child presented with his mother complaining of pain with absent of any radiographic pathosis. In the 6 months follow up periapical lesion was developed. The second case show redness and swelling in the soft tissue around the tooth with periapical radiolucency in the radiograph in the 6 months follow up.

As a comparison between Biodentine and MTA groups in the clinical and radiographic outcome, the difference between both groups was statistically non-significant as indicated by Chi square test $(p=0.7646>0.05)$.

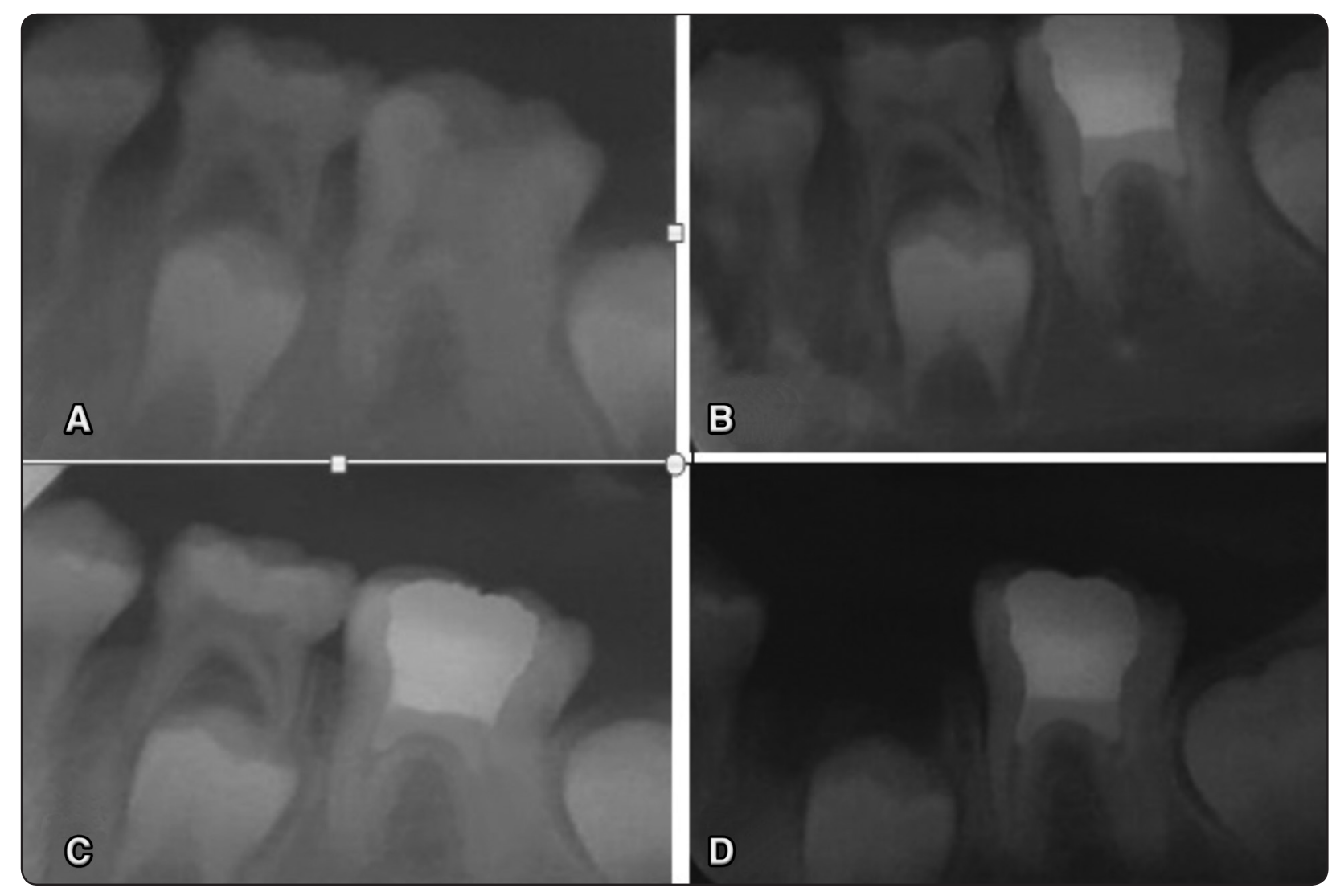

Fig. (1) Show Biodentine radiograph A) preoperative radiograph, B) postoperative radiograph after 6 weeks, C) postoperative radiograph after 6 months, D) postoperative radiograph after 12 months 


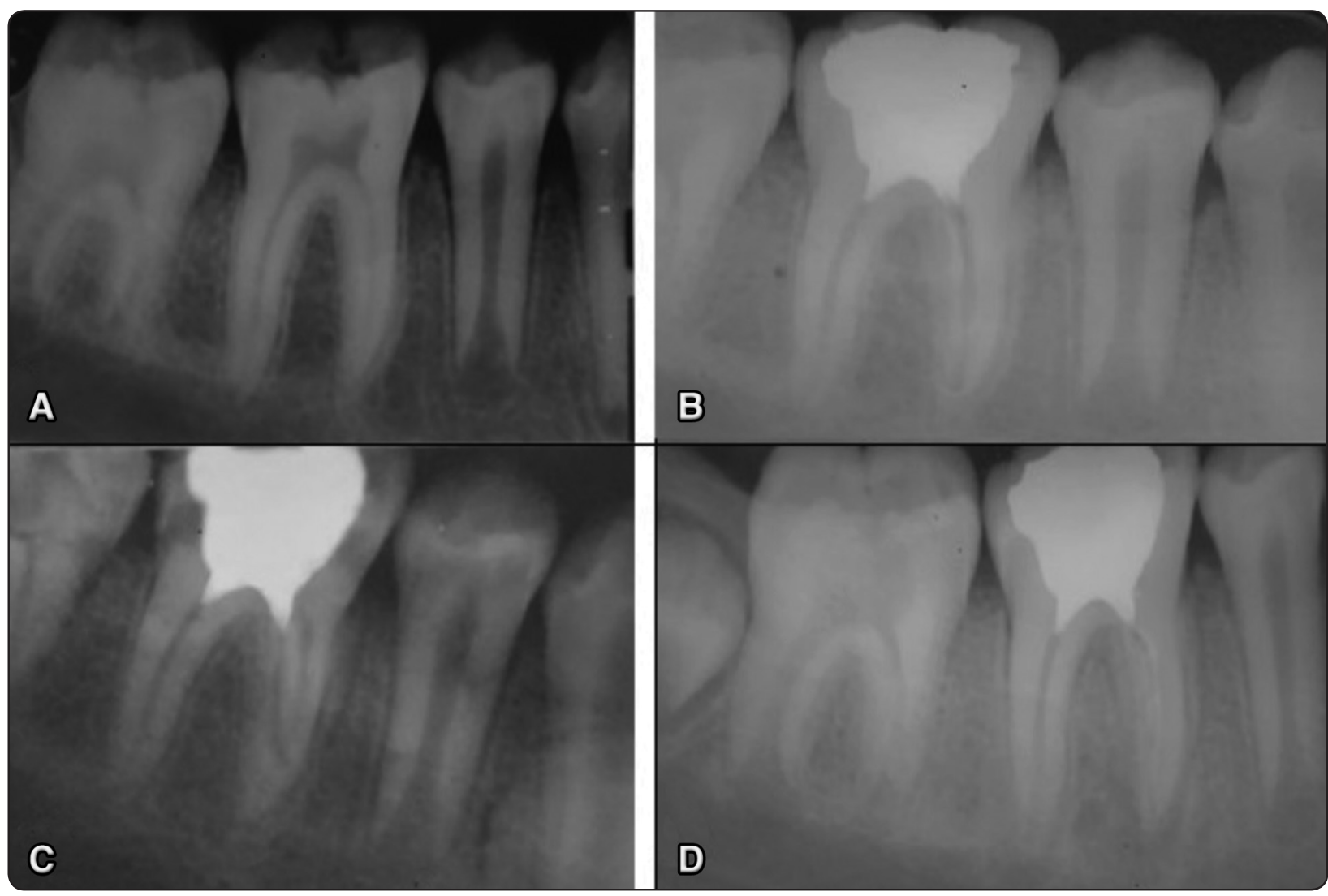

Fig. (2) Show ENDOCEM Zr (MTA) radiograph A) preoperative radiograph, B) postoperative radiograph after 6 weeks, C) postoperative radiograph after 6 months, D) postoperative radiograph after 12 months.

TABLE (2) show the clinical follow up period of Biodentine and ENDOCEM $\operatorname{Zr}$ ( (MTA)

\begin{tabular}{|c|c|c|c|c|c|c|}
\hline \multirow{2}{*}{} & \multicolumn{3}{|c|}{ Biodentine } & \multicolumn{2}{c|}{ MTA } \\
\cline { 2 - 7 } & 6 weeks & 6 months & 1 year & 6 weeks & 6 months & 1 year \\
\hline Success & $20(100 \%)$ & $19(95 \%)$ & $19(95 \%)$ & $19(95 \%)$ & $18(90 \%)$ & $18(90 \%)$ \\
\hline Failure & $0(0 \%)$ & $1(5 \%)$ & $1(5 \%)$ & $1(5 \%)$ & $2(10 \%)$ & $2(10 \%)$ \\
\hline
\end{tabular}

TABLE (3 ) Show radiographic of follow up period of Biodentine and ENDOCEM Zr ((MTA)

\begin{tabular}{|c|c|c|c|c|c|c|}
\hline & \multicolumn{3}{|c|}{ Biodentine } & \multicolumn{2}{c|}{ MTA } \\
\cline { 2 - 7 } & 6 weeks & 6 months & 1 year & 6 weeks & 6 months & 1 year \\
\hline Success & $20(100 \%)$ & $19(95 \%)$ & $19(95 \%)$ & $20(100 \%)$ & $18(90 \%)$ & $18(90 \%)$ \\
\hline Failure & $0(0 \%)$ & $1(5 \%)$ & $1(5 \%)$ & $0(0 \%)$ & $2(10 \%)$ & $2(10 \%)$ \\
\hline
\end{tabular}




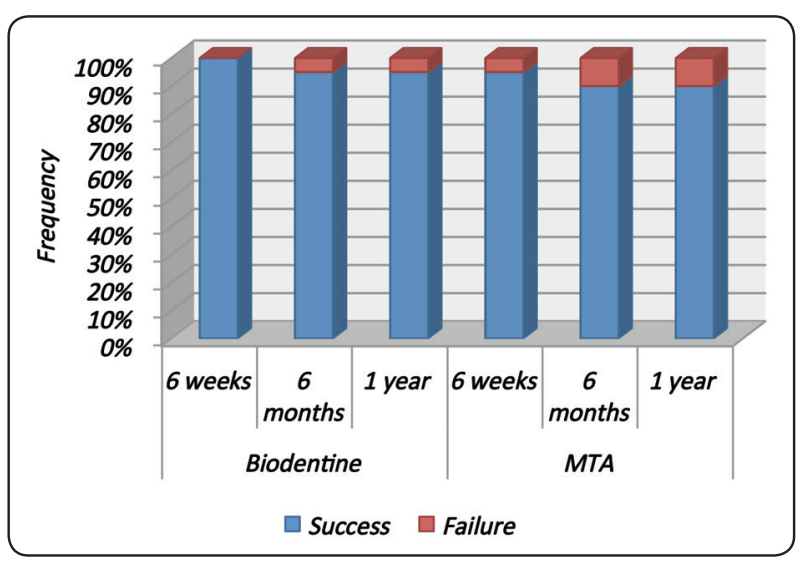

Fig (3) Show clinical follow up period of Biodentine and ENDOCEM Zr (MTA)

\section{DISCUSSION}

Several studies support the concept that pulpal inflammation can be confined to the area next to the carious exposure and not extending far from 2 $\mathrm{mm}$ away from the exposure site, radicular pulp was rarely inflamed. Therefore, if the inflamed tissue is removed and the pulp is dressed with suitable material, the conservation of a remaining healthy pulp is potentially possible $e^{5,11,18}$. The purpose of vital pulpotomy in permanent teeth in children is to treat reversible pulpal injuries and maintain radicular pulp vital and healthy, and therefore preserve the tooth in a viable condition ${ }^{1}$.

Many factors have been affecting the success of vital pulptomy. One of the most important factors is the condition of root apex. High success rate for vital pulp therapy in young permanent teeth with open root apices was reported by many studies. Nyerere J.W. et al (2006) ${ }^{19}$ demonstrated a success rate of $100 \%$ for premolars and $97.1 \%$ for molars in alleviating acute pain after performing vital pulpotomy in molars or premolars with open apex using zinc oxide eugenol as the material of choice. Qudeimat M.A. et al (2007) ${ }^{20}$ reported up to $93 \%$ success rate for vital pulp therapy in young permanent teeth with open apices. Also Nosrat A. et al (2013) $)^{21}$ showed almost $100 \%$ of pulp vitality with continuation of root development after 12

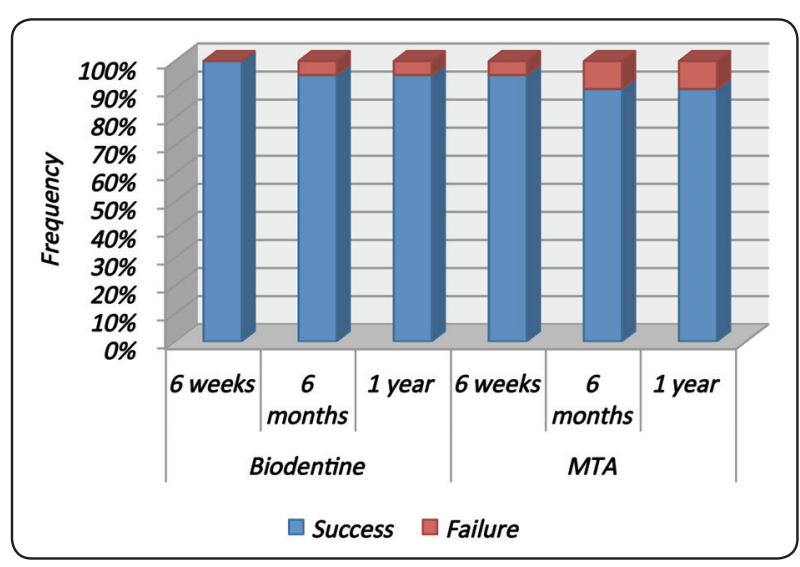

Fig. (4) Show radiographic of follow up period of Biodentine and ENDOCEM Zr ((MTA)

months of using MTA and $\mathrm{Ca}(\mathrm{OH})$ in immature molars with clinical carious exposure.

Controversies still exist in the literature about the outcome of pulpotomy for cariously exposed pulp in mature permanent teeth and it has been investigated to a much lesser extent but with high success rate. Barngkgei I.H. et al $(2013)^{10}$ reported $100 \%$ clinical and radiographic success rate of pulpotomy treatment with MTA in symptomatic mature permanent teeth. Also Aguilar P. and Linsuwanont P. (2011) $)^{22}$ have demonstrated a relatively high success rate of $99.4 \%$ for partial pulpotomy and $99.3 \%$ for full pulpotomy in vital permanent teeth with closed apices.

The impact of age has direct effect on the success rate of VPT. Several studies recommended the use of vital pulp therapy in young patients, because older pulps are fibrous and less cellular with limited blood supply. ${ }^{23,24}$ The younger the pulp the higher success rate may achieved ${ }^{2}$. Accordingly in this study pulpotomy was planned for all the patients with age ranges between 10 and 13 years to achieve a predictable outcome.

Hemorrhage from the exposed pulp tissue is due to the inflammatory response of the pulp to bacteria and their by-products. Proper hemostasis is the second key to increase the success rate of vital pulp therapy because the blood clots that remain 
at the pulp material interface leads to treatment failure $^{5}$. The ideal solution for hemostasis is sodium hypochloride because it helps in rapidly controlling the hemorrhage, disinfecting the cavity, removing the coagulum and dentin chips and aiding in formation of Dentin Bridge ${ }^{25}$. In the current study we use $3 \% \mathrm{Naocl}$ as a hemostatic agent to insure proper controlling of hemorrhage.

Coronal seal is another important factor in predicting pulpal responses to VPT. Hence, in the present study, double seal provided by either MTA or Biodentine and composite restoration was done to insures ensures a tight coronal seal.

Proper dressing material is another important factor in the success of vital pulp therapy. Pulp covering material should be biocompatible, noncytotoxic, and antibacterial ${ }^{5}$. Over the course of time, several materials have been used as pulp capping agents. MTA is considered to be a suitable dressing material in vital pulp therapy procedures due to its biocompatibility and improved sealing ability $^{2}$. Endocem is the next new generation of MTA with high physical properties which enhance less discoloration and improve the esthetics of the tooth; it has excellent sealing property and biocompatibility. With great handling properties for pulpotomy therapy of permanent teeth such as fast setting time and radiopacity. It is the safest MTA that exhibits low grade of calcification of pulp chambers and root canals even long after partial pulpotomy therapy ${ }^{14}$.

The result of this study showed that after a period of 6 weeks, 6months and 1 year follow up, the clinical success rates for the ENDOCEM $\mathrm{Zr}$ was $95 \%$ $.90 \%$ and $90 \%$, respectively and the radiographic success rates was $100 \%, 90 \%$ and $90 \%$ respectively. Barngkgei I.H. et al $(2013)^{10}$ revealed up to $100 \%$ clinical and radiographic success with the use of MTA in the patient aged 27-54 years with up to 24-42 months follow up. Alqaderi H. E.et al (2014) used MTA as a pulpotomy capping agent in mature permanent teeth of young patient (10 to 15years) with follow-up period of 25 months ${ }^{1}$. The estimated success rate of clinical and radiographic evidence was $90 \%$. Taha N. A. et al $(2015)^{11}$ assess the use of mineral trioxide aggregate in pulpotomy treatment of cariously exposed permanent teeth in 11-51 year patient with up to 3 years follow up. The success rate of the cases was $100 \%$ clinical and $97.5 \%$ radiographic success during the first year, and $92.7 \%$ at 3 years. However, to date, only a few studies have investigated the biocompatibility and physical properties of Endocem in vitro ${ }^{7,18,25}$, Song M. et al (2015) evaluate and compare the short-term clinical outcomes of direct pulp capping of permanent teeth with Endocem and Pro- Root MTA. Endocem show success rate higher than Pro-Root MTA ${ }^{14}$.

New tricalcium silicate cements (biodentine) are of interest in the treatment of cases of pulp capping and pulpotomy as it may simulate the dentine structure in its mechanical properties. In the current study, the clinical and radiographic success rate of biodentine pulpotomy in 6 weaks, 6months and 1 year follow up was 100\%,95\% and $95 \%$ respectively. Few researches were done to investigate the effect of biodentine as a capping material in immature and mature teeth; most of these researches were case report. Villat C. et al. (2013) $)^{15}$ performed partial pulpotomy using Biodentine in an immature second right mandibular premolar. At both 3 and 6 months follow up, preservation of tooth vitality was noticed in the whole follow up period, and adequate dentinbridge formation in the pulp chamber was found in the radiographic examination. Also it demonstrated a fast tissue response both at the pulpal and root dentin levels. Ree M. (2014) ${ }^{26}$ use Biodentine ${ }^{\mathrm{TM}}$ as a pulp capping material in an pulpally involved immature tooth in 2 cases. In both cases, treatment provided removal of symptoms and continuation of root formation with no signs of discoloration after 18 months follow up. Borkar S. A. and Ataide I. $(2015)^{17}$ report four cases of traumatized, matured, maxillary permanent central incisors, which have been treated by Biodentine pulpotomy, the teeth were assessed clinically and radiographically for 
periapical healing. Absent of spontaneous pain was noticed; with preservation of tooth vitality and absence of periapical radiolucency after 18 months.

As a comparison between Biodentine and MTA groups in the clinical and radiographic outcome, the result of this study revealed statistically insignificant difference between both groups ( $p=0.7646>0.05$ ). The use of ENDOCEM Zr as new generation of MTA overcomes the drawbacks of the conventional MTA. This may be the main reason for insignificant difference that found between biodentine and MTA.

Many studies compare the response of the pulpdentin complex in human permanent molars after direct capping when using biodentine with that of MTA. In 2013 Nowicka A. et $\mathrm{al}^{27}$ publish an in vitro study which compare between biodentine and MTA as a direct pulp capping materials. He found that no significant differences between the Biodentine and MTA, both groups have complete dentinal bridge formation and an absence of pulpal inflammation. In 2015 he publish another in vivo research in which he made direct pulp capping in Forty-four cariesfree, human third molars needed to be extracted using calcium hydroxide, MTA, Biodentine, or Single Bond Universal. The teeth were extracted after 6 weeks, and cone-beam tomographic imaging and histologic examination were done for all the spacimen. He found that in the Biodentine group the dentin bridges have the highest volumes ${ }^{28}$.

Also De Rossi A.etal (2014) $)^{29}$ made radiographic, histopathologic, and histomicrobiological analyses to compare the pulpal responses of dogs' teeth after pulpotomy with (Biodentine) and (MTA) . He found that Biodentine has a higher tissue compatibility and inhance dentine bridge formation after pulpotomy in all specimens than those formed with use of MTA. Radiographically, mineralized tissue bridge formation was observed in more specimens treated with Biodentine (96.8\%) than with MTA (72.2\%) $(\mathrm{P}=.02)$. Daltoé M.O. et al $(2016)^{30}$ compared the cell viability of dental pulp cells treated with Biodentine and mineral trioxide aggregate (MTA) after pulpotomy in a specimens of teeth from dogs. He concluded that no significant differences were found between MTA and Biodentine regarding the cell viability.

Biodentine have superior properties than coventional MTA. It had higher flexural strength, flexural modulus and compressive strength ${ }^{31}$, it has been demonstrated to have bioactive properties, encourages hard tissue regeneration so it was suitable to be used as a pulp capping agent. The material had improved marginal integrity as it allow for the formation of hydroxyapatite crystals at the surface which improved its sealing ability ${ }^{17,26}$. Biodentine have shorter setting time than MTA, According to Parirokh M. and Torabinejad M. (2010), it can take up to 3 hours for MTA to set . while biodentine material sets in 12 minutes due to presence of calcium chloride accelerator and decreasing the particles size as well as the water content of the system. Biodentine can be applied on dentin without using any conditioning agent, and did not cause staining of dentin as has been reported with MTA. In general, bioceramic materials have better clinical handling properties $^{2,33}$.

\section{CONCLUSION}

In this randomized controlled study, Biodentine has clinical and radiograghic success rate comparable to ENDOCEM Zr as a vital pulpotomy in mature permanent molars with carious exposures.

\section{REFERENCE}

1. Alqaderi H. E., Al-Mutawa S. A. and Qudeimat M. A. MTA pulpotomy as an alternative to root canal treatment in children's permanent teeth in a dental public health setting. Journal of dentistry. 2014; 42(11):1390-1395.

2. Solomon R. V., Faizuddin U., Karunakar P., Sarvani G. D. and Soumya S. S. Coronal Pulpotomy Technique Analysis as an Alternative to Pulpectomy for Preserving the Tooth Vitality, in the Context of Tissue Regeneration: A Correlated Clinical Study across 4 Adult Permanent Molars. Case Rep.Dentist. 2015; (2015):1-12. 
3. Sloan A.J. and Waddington R.J. Dental pulp stem cells: what, where, how? International Journal of Paediatric Dentistry. $2009 ; 19: 61-70$.

4. Alqaderi H., Lee C., Borzangy S., Pagonis T.C. Coronal pulpotomy for cariously exposed permanent posterior teeth with closed apices: A systematic review and metaanalysis. Journal of Dentistry. 2016; 44 :1-7.

5. Ghoddusia J., Forghania M. and Parisay I.New Approaches in Vital Pulp Therapy in Permanent Teeth. IEJ. 2014; 9(1):15-22.

6. Asgary S, and Ehsan S. Permanent molar pulpotomy with a new endodontic cement: A case series. J Conserv Dent. 2009, Jan-Mar; 12(1): 31-36.

7. Witherspoon D. E. Vital pulp therapy with new materials: new directions and treatment perspectives-permanent teeth. Pediatr Dent 2008; 30:220-224.

8. Camilleri J. The chemical composition of mineral trioxide aggregate. J Conserv Dent. 2008, Oct; 11(4):141-3.

9. Colorado C. Clinical and Radiographic Evaluation of MTA Pulpotomies: A Retrospective Study. MS thesis,Virginia Commonwealth University. 2014.

10. Barngkgei I. H., Halboub E. S. and Alboni R. S. Pulpotomy of symptomatic permanent teeth with carious exposure using mineral trioxide aggregate. Iranian Endodontic Journal. 2013; 8:65-8.

11. Taha N. A., Ahmad M. B. and Ghanim A. Assessment of Mineral Trioxide Aggregate pulpotomy in mature permanent teeth with carious exposures. International Endodontic Journal. 2015; 36(3):225-31

12. Witherspoon D.E., Small J.C. and Harris G.Z. Mineral trioxide aggregate pulpotomies: A case series outcomes assessment. J Am Dent Assoc. 2006; 137: 610-8.

13. Ravichandra P. V., Jayaprada Reddy S., Harikumar V. and Kavita A. Mineral Trioxide Aggregate. IJDA. 2011; JulySeptember 3:3.

14. Song M., Kang M., Kim H.C. and Kim E. A randomized controlled study of the use of Proroot Mineral Trioxide Aggregate and Endocem as direct pulp capping materials. J Endod. 2015,Jan; 41 (1):11-5.

15. Villat C., Grosgogeat B, Seux D. and Farge P. Conservative approach of a symptomatic carious immature permanent tooth using a tricalcium silicate cement (Biodentine): a case report. Restor Dent Endod. 2013, Nov; 38(4): 258-262.
16. Da Fonseca T. S., da Silva G. F., Tanomaru-Filho M. and Sasso-Cerri E. In vivo evaluation of the inflammatory response and IL-6 immunoexpression promoted by Biodentine and MTA Angelus. International indodontic journal; 49 (2): 145-153.

17. Borkar S. A. and Ataide I. 2015.Biodentine pulpotomy several days after pulp exposure: Four case reports.J Conserv Dent. 2016, Jan-Feb; 18(1): 73-78.

18. Langeland K.Tissue response to dental caries. Endod Dent Traumatol. 1987; 3 (4):149-71.

19. Nyerere J. W., Matee M. I. and Simon E. N. M. "Emergency pulpotomy in relieving acute dental pain among Tanzanian patients," BMC Oral Health.2006; 6: 1.

20. Qudeimat M. A., Barrieshi-Nusair K. M. and Owais A. I. Calcium hydroxide vs mineral trioxide aggregates for partial pulpotomy of permanent molars with deep caries. Eur Arch Paediatr Dent. 2007, Jun; 8 (2):99-104.

21. Nosrat A., Seifi A. and Asgary S.Pulpotomy in caries-exposed immature permanent molars using calcium-enriched mixture cement or mineral trioxide aggregate: a randomized clinical trial. Int J Paediatr Dent. 2013, Jan; 23(1): 56-63.

22. Aguilar P. and Linsuwanont P.Vital pulp therapy in vital permanent teeth with cariously exposed pulp: a systematic review. Journal of Endodontics. 2011; 37 (5):581-587.

23. Ward J.Vital pulp therapy in cariously exposed permanent teeth and its limitations. Aust Endod J. 2002; 28(1):29-37.

24. Matsuzaka K., Muramatsu T., Katakura A., Ishihara K., Hashimoto S., Yoshinari M., et al.Changes in the homeostatic mechanism of dental pulp with age: expression of the corebinding factor alpha-1, dentin sialoprotein, vascular endothelial growth factor, and heat shock protein 27 messenger RNAs. Journal of Endodontics. 2008; 34:818-21.

25. Demir T. and Cehreli Z.C.Clinical and radiographic evaluation of adhesive pulp capping in primary molars following hemostasis with $1.25 \%$ sodium hypochlorite: 2-year results. Am J Dent. 2007; 20 (3):182-8.

26. Ree M.Vital pulp therapy with Biodentine ${ }^{\mathrm{TM}}$ in two immature, traumatized teeth. INTERNATIONAL DENTISTRY - AFRICAN EDITION. 2014; 5(2) 6-13.

27. Nowicka A., Lipski M., Parafiniuk M., Sporniak-Tutak K., Lichota D., Kosierkiewicz A., Kaczmarek W. and Buczkowska-Radlińska J. Response of human dental pulp capped with biodentine and mineral trioxide aggregate.J Endod. 2013, Jun; 39(6):743-7. 
28. Nowicka A., Wilk G., Lipski M., Kołecki J. and Buczkowska-Radlińska J. Tomographic Evaluation of Reparative Dentin Formation after Direct Pulp Capping with $\mathrm{Ca}(\mathrm{OH}) 2$, MTA, Biodentine, and Dentin Bonding System in Human Teeth. J Endod. 2015, Aug; 41(8):1234-40.

29. De Rossi A., Silva L.A., Gatón-Hernández P., Sousa-Neto M. D., Nelson-Filho P., Silva R. A. and de Queiroz A. M. Comparison of pulpal responses to pulpotomy and pulp capping with biodentine and mineral trioxide aggregate in dogs. J Endod. 2014,Sep; 40(9):1362-9.

30. Daltoé M. O., Paula-Silva F. W., Faccioli L. H., GatónHernández P. M., De Rossi A. and Bezerra Silva L. A. Expression of Mineralization Markers during Pulp Response to Biodentine and Mineral Trioxide Aggregate. J Endod. 2016, Apr; 42(4):596-603.
31. Natale L.C., Rodrigues M.C., Xavier T.A., Simões A., de Souza D.N. and Braga R.R. Ion release and mechanical properties of calcium silicate and calcium hydroxide materials used for pulp capping. Int Endod J. 2015 Jan;48(1):89-94

32. Parirokh M. and Torabinejad M. Mineral Trioxide Aggregate: A Comprehensive Literature Review-Part I: Chemical, Physical, and Antibacterial Properties. J Endod. 2010;36:16-27.

33. Percinoto C., de Castro A. M.and Pinto L.M. Clinical and radiographic evaluation of pulpotomies employing calcium hydroxide and trioxide mineral aggregate. Gen Dent. 2006; 54: 258-261. 